\title{
Knowledge, attitude and practices regarding food hygiene and sanitation of food handlers in Kuala Pilah, Malaysia.
}

\begin{abstract}
This study evaluates the knowledge, attitude and practices (KAP) of food handlers working in restaurants in Kuala Pilah, Malaysia. Data were collected in 2010 involving 64 food handlers. The results show that the food handlers have excellent knowledge and attitude, and good practices toward food hygiene with mean score (SD) of 83.98 (13.26), 82.8 (10.94) and 77.04 (14.98), respectively. There is significant correlation between educational level and attitude $(\mathrm{p}=.008)$, knowledge and attitude $(\mathrm{p} \leq .001)$, knowledge and practice $(\mathrm{p}=.007)$ and attitude and practice $(\mathrm{p}=.041)$. Even though the result shows satisfactory KAP levels of the food handlers, some aspects on hygiene measures like refreezing food item, clean working area and wearing of jewelry and watch need to be emphasized.
\end{abstract}

Keyword: Food handlers; Knowledge; Attitudes; Practices. 